\title{
Stretchable electrothermochromic fibers based on hierarchical porous structures with electrically conductive dual-pathways
}

\author{
Hongwei Fan ${ }^{1}$, Qiang $\mathrm{Li}^{1}$, Kerui $\mathrm{Li}^{1,2^{*}}$, Chengyi Hou ${ }^{1}$, Qinghong Zhang ${ }^{3}$, Yaogang $\mathrm{Li}^{3}$ and \\ Hongzhi Wang ${ }^{1 *}$
}

\begin{abstract}
Stretchable color-changing fibers are urgently demanded for smart textiles/clothing due to their perfect implantability, permeability of vapor and heat, and flexibility/ stretchability. Herein, stretchable electrothermochromic fibers were fabricated with unconventional stretchable conductive fibers as core layers and thermochromic coatings as shell layers. In the stretchable conductive fibers, hierarchical porous structures with percolative one-dimensional (1D) conductive networks were constructed through phase inversion of carbon nanotube/polyurethane (CNT/PU) solutions. With the deposition of silver nanoparticles (AgNPs) on the surface of micro-pores, electrically conductive dual-pathways consisting of 0D AgNPs and 1D CNTs were formed to significantly enhance the electric conductivity and thus improve the electrothermal performance of the fibers. More importantly, because of the connective CNTs and AgNPs, such dual-pathways ensured the electron transport under the stretching state, preventing the sharp decay of conductivity and electrothermal performance. Through the continuous wet-spinning method, the stretchable conductive fibers can be easily obtained with the length up to several meters. At last, stretchable electrothermochromic fibers were prepared with two color-changing modes and implanted into textile perfectly, advancing their applications in wearable display and military adaptive camouflage of smart clothing.
\end{abstract}

Keywords: hierarchical porous structures, conductive dualpathways, stretchable conductive fibers, electrothermochromic fibers, smart clothing

\section{INTRODUCTION}

Fibers, as the basic building blocks of clothing and tex- tiles, have been closely related to our modern society. As one of the most important functionalities, fiber colors are indispensable and have been widely utilized in people's daily life [1-4]. In recent years, smart fiber and textiles have attracted wide attention with the increasing popularity of wearable electronic products and the rise of the smart clothing concept [5-11]. The smart color-changing fibers as an important branch of smart fibers have attracted extensive interest due to their promising applications in wearable displays, visual sensing and adaptive camouflage [12-16]. However, the colors of existing commercial fibers can not be changed on demand due to the passive optical properties of traditional dyes $[17,18]$. Therefore, the controllable color-changing fibers are urgently needed to realize various smart applications [1923].

Recently, various color-changing fibers were reported in response to different stimuli, such as electricity, light and magnetism [24-28]. For example, Shang et al. [29] prepared the structural colored fibers by embedding ethylene glycol droplets containing $\mathrm{Fe}_{3} \mathrm{O}_{4} @ \mathrm{C}$ nanoparticles into polydimethylsiloxane. Under the magnetic field, the $\mathrm{Fe}_{3} \mathrm{O}_{4} @ \mathrm{C}$ nanoparticles formed the chain-like structure to realize the color change of fibers from brown to yellow-green. Additionally, Pinto and colleagues [30] prepared the photochromic polypropylene (PP) fibers based on ultraviolet-responsive silica@phosphomolybdate nanoparticles through melt spinning technology and achieved color changes from yellow-green to blue. However, these fibers showed an unavoidable limitation in controllability because these stimuli are easily influ-

\footnotetext{
${ }^{1}$ State Key Laboratory for Modification of Chemical Fibers and Polymer Materials, College of Materials Science and Engineering, Donghua University, Shanghai 201620, China

${ }^{2}$ Department of Chemical and Biomolecular Engineering, National University of Singapore, Singapore 117585, Singapore

${ }^{3}$ Engineering Research Center of Advanced Glasses Manufacturing Technology, Ministry of Education, Donghua University, Shanghai 201620, China * Corresponding authors (emails: chelik@nus.edu.sg (Li K); wanghz@dhu.edu.cn (Wang H))
} 
enced by the surrounding environment.

Compared with these stimuli sources, electricity is an efficient and controllable means to realize color changes [31]. For example, our group [32] prepared electrochromic (EC) fibers based on a helically-twined metal electrode structure before, and successfully obtained multiple color changes by using three types of EC conjugated polymers. Despite these, limited by their complex multilayer structures and vulnerable electrolytes, stretchability, working stability and scalable preparation of EC fibers are difficult to achieve [33,34]. Therefore, smart color-changing fibers should be further developed to satisfy several requirements: first, the fiber color should be easily controlled and tuned. Second, the fibers should be stretchable to ensure the wearing comfortability under human locomotion and joint bending. Lastly, scalable production is highly needed for the practical applications of smart fibers. Compared with the color-changing fibers above, the electrothermochromic fibers show promising potential to satisfy the requirements due to their relatively simple structures and great color controllability through electricity stimuli.

To achieve the stretchable electrothermochromic fibers, the stretchable conductive fibers are crucial. Herein, the stretchable conductive fibers were first prepared with hierarchical porous structures through the phase inversion method. With the chemical reduction of silver precursors on the surface of polyurethane (PU) micro-pores, electrically conductive dual-pathways were formed consisting of zero-dimensional (0D) silver nanoparticles (AgNPs) and 1D carbon nanotubes (CNTs) in PU matrix. Because of the formation of conductive dual-pathways, the conductivity of fibers was highly improved, which was able to enhance the electrothermal performance of the stretchable fibers. In addition, contributed by the coupling of interconnected CNTs and the AgNPs, two types of conductive paths, stretchable conductive fibers showed high conductivity under stretching states and great tensile stability during stretching/releasing. Through the continuous wet-spinning method, the stretchable conductive fibers can be easily obtained with the length up to several meters. At last, long-range stretchable electrothermochromic fibers capable of multiple color changes under different strains or different voltages were prepared to implant into textile perfectly, demonstrating their promising applications in smart clothing.

\section{EXPERIMENTAL SECTION}

Carbon nanotubes (CNTs, multi-walled type and socalled multi-walled CNT, Chengdu Organic Chemistry
Co., Ltd.), PU powders (BASF Co., Ltd.), and thermochromic inks (Shenzhen Qiansebian Pigments Co., Ltd.) were commercially available and used without further purification. Silver trifluoroacetate (98\%), hydrazine hydrate $\left(\mathrm{H}_{4} \mathrm{~N}_{2}, 50 \%\right)$, and $\mathrm{N}, \mathrm{N}$-dimethylformamide (DMF, 99.5\%) were purchased from Sinopharm Chemical Reagent Co., Ltd.

\section{Preparation of the stretchable conductive fibers}

The CNTs with contents of $1 \mathrm{wt} \%, 5 \mathrm{wt} \%, 10 \mathrm{wt} \%$ and $20 \mathrm{wt} \%$, respectively, were dispersed in $100 \mathrm{~mL}$ of DMF by ultrasonic treatment for $6 \mathrm{~h}$. Then, $18.9 \mathrm{~g}$ of PU was added into the CNT dispersions slowly and continuously stirred at $70^{\circ} \mathrm{C}$ to form $\mathrm{CNT} / \mathrm{PU}$ dispersions. The dispersions were then wet-spun using a syringe with a spinning speed of $5 \mathrm{~mL} \mathrm{~h}^{-1}$ in a water bath containing $4 \mathrm{wt} \% \mathrm{CaCl}_{2}$ to obtain the porous CNT/PU fibers. After that, the porous CNT/PU fibers were directly transferred to the Ag-ion precursor solution bath containing silver trifluoroacetate $\left(10 \mathrm{mg} \mathrm{mL}^{-1}\right)$ in ethanol solution and maintained for $30 \mathrm{~min}$. After being dried at room temperature, they were soaked in $\mathrm{H}_{4} \mathrm{~N}_{2}$ aqueous solution for 30 min to reduce Ag ions for the deposition of AgNPs in the micro-pores of CNT/PU fibers. The CNT/PU fibers deposited with AgNPs were transferred in ethanol and deionized water to wash the excess residue. At last, after being air-dried, the stretchable conductive fibers were obtained.

\section{Preparation of the stretchable electrothermochromic fibers} The "base" and "curing agent" of Sylgard 184 (Dow Corning) were mixed at a ratio of 10:1 and solidified in the outer layer of the stretchable conductive fibers as the protective layer. The red thermochromic inks (color switching from red to white at $38^{\circ} \mathrm{C}$ ) and blue thermochromic inks (color switching from blue to white at $45^{\circ} \mathrm{C}$ ) were mixed with a weight ratio of $5: 2$. It should be mentioned that if needed, other thermochromic inks with different color changes or switching temperatures can be custom-designed. The stretchable conductive fibers were immersed into the mixed thermochromic inks and then transferred in an oven at $60^{\circ} \mathrm{C}$ to evaporate the solvent of coated inks to obtain the stretchable electrothermochromic fibers.

\section{Characterization and measurements}

The morphologies and microstructures of the fibers were characterized via field emission scanning electron microscopy (FE-SEM; SU8000, Hitachi, Japan) and SEM (Phenom-Word BV, G2pro). The thermal images were 
taken by a thermal imaging camera (FLIR A300-Serious, Sweden). The mechanical property was measured using the Instron testing system (INSTRON 5969, Instron Corporation, USA). A mechanical motor (homemade device) was used for stretching/releasing fibers. The electrochemical workstation (CHI760D, Shanghai Chenhua Instruments, China) was used to measure the resistance variations of the stretchable conductive fibers upon stretching/releasing cycles. The reflectance spectra of the stretchable electrothermochromic fibers were obtained using a fiber-optic spectrometer (PG2000-Pro-Ex, Ideaoptics Technology Co., Ltd., China). The wavelengths giving the maximum reflectance difference between different colors were selected to calculate the reflectance contrasts $(\Delta \mathrm{RT})$ of the stretchable electrothermochromic fibers. The frictional cycles of the stretchable electrothermochromic fibers were measured through a fabric abrasion tester (YG401E, Ningbo Textile Instrument Factory, China).

\section{RESULTS AND DISCUSSION}

As shown in Fig. 1a, the stretchable electrothermochromic fiber was prepared through wet spinning, deposition of AgNPs and coating with thermochromic layers. Briefly, the porous composite fibers consisting of CNTs and PU were prepared by the phase inversion of $\mathrm{CNT} / \mathrm{PU}$ solutions. In the PU matrix, percolative CNT networks were constructed for 1D conductive pathways. After deposition of AgNPs through chemical reduction of
Ag-ion precursor, AgNPs were coated on the surface of micro-pores of CNT/PU composite fibers, leading to the formation of the second conductive pathways consisting of $0 \mathrm{D}$ AgNPs. Contributed by the electrically conductive dual-pathways consisting of surficial 0D AgNPs and embedded 1D CNTs in PU micro-pores, the fabricated stretchable conductive fibers showed highly improved conductivity, high conductivity retention under stretching state, and great tensile stability during stretching/ releasing cycles. Finally, after the coating of the PDMS layer and thermochromic inks on the stretchable conductive fibers, the stretchable electrothermochromic fibers were obtained with porous conductive fibers as core layers and thermochromic ink as shell layers (Fig. 1b). Because of the thin PDMS layer (ca. $40 \mu \mathrm{m}$ ), the thermal transfer was hardly influenced between the conductive fiber and thermochromic coatings (see detailed description in Fig. S1). Additionally, it should be mentioned that if needed, other thermochromic inks with different color changes or switching temperatures can be custom-designed.

As shown in Fig. 1c, the stretchable electrothermochromic fiber with a diameter of ca. $0.6 \mathrm{~mm}$ showed no interfacial delamination, indicating good adhesion between each layer. Moreover, obvious hierarchical porous and multilayer structure were observed. The hierarchical porous structure was caused by phase separation from the homogeneous solution phase of PU in the DMF solvent to the solid phase of PU in the water/DMF mixed solu-
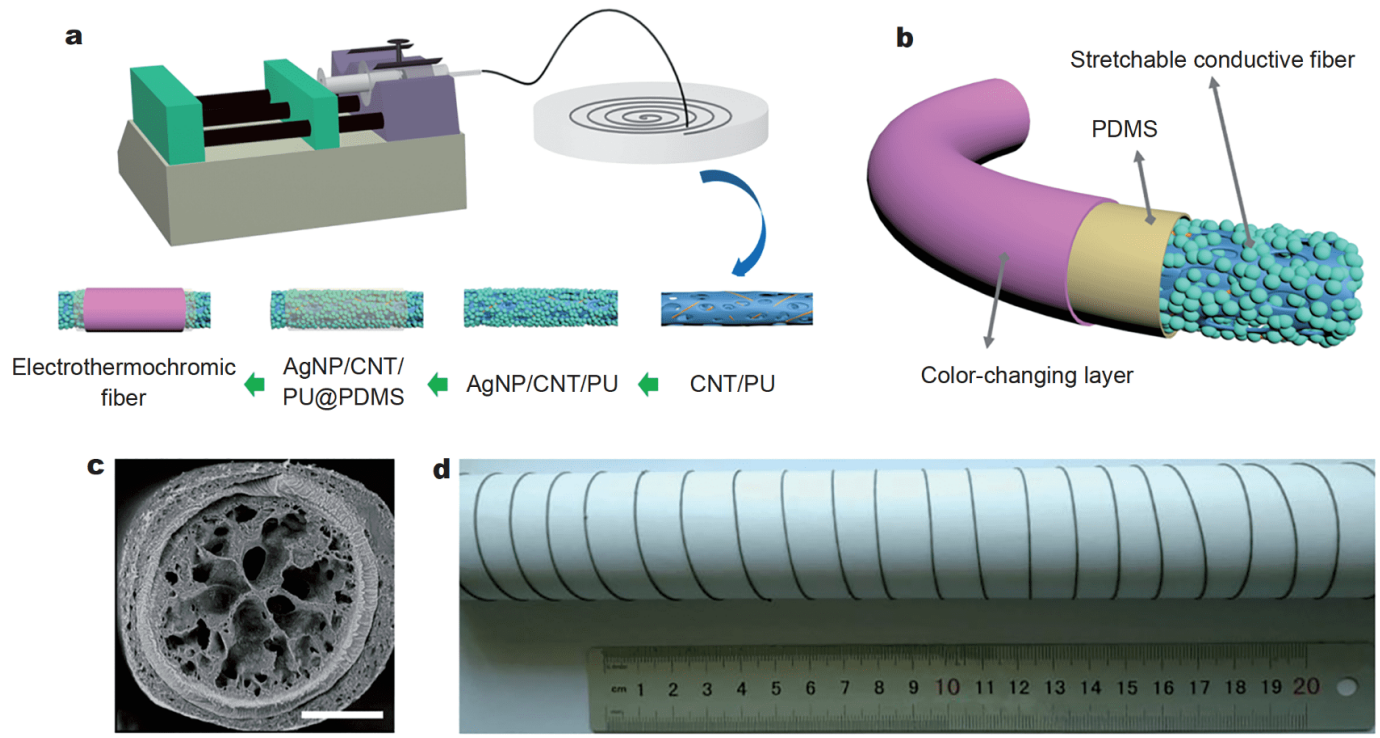

Figure 1 Fabrication of the stretchable electrothermochromic fibers. Schematic illustration of (a) preparation process and (b) structure of the stretchable electrothermochromic fiber. (c) Cross-sectional SEM image of the stretchable electrothermochromic fiber (scale bar: $200 \mu \mathrm{m}$ ). (d) Digital photograph of the stretchable conductive fiber with a length of $3.0 \mathrm{~m}$. 
tion due to the diffusion and intrusion of water from the water bath [35]. As shown in Fig. 1d, because of the facile fabrication process, a 3-m-long porous stretchable conductive fiber was successfully obtained and collected on a cylindrical spool. In addition, the electrothermochromic fiber showed great stretchability and did not break down under a $60 \%$ strain (Fig. S2).

We first increased the loadings of CNTs, the conductive fillers, in the composite fibers for optimizing CNT loadings through the investigation of mechanical performance as well as increasing the conductivity of fibers. As shown in Fig. 2a, as the loading of CNTs increased from 0, 1, 5, 10 to $20 \mathrm{wt} \%$, the elongation at break of the porous CNT/ PU composite fibers decreased from $358 \%$ for $0 \mathrm{wt} \%$ CNT loading, $279 \%$ for $1 \mathrm{wt} \%$ CNT loading, $123 \%$ for $5 \mathrm{wt} \%$ CNT loading, $57.3 \%$ for $10 \mathrm{wt} \%$ CNT loading, to $8.1 \%$ for $20 \mathrm{wt} \% \mathrm{CNT}$ loading. The fracture stresses were also reduced from $2.16,2.19,1.22,0.85$ to $0.38 \mathrm{MPa}$. Therefore, after comprehensively considering the mechanical performance and conductivity, the porous CNT/ PU composite fibers with the CNT loading of $5 \mathrm{wt} \%$ and the conductivity of $0.175 \mathrm{~S} \mathrm{~cm}^{-1}$ were selected for the following study and usage.

In addition, the morphologies of porous CNT/PU composite fibers were investigated, as shown in Fig. 2b, c and Fig. S3. The porous CNT/PU composite fiber exhibited the hierarchical porous structure consisting of larger-sized micro-pores ranging from 100 to $200 \mu \mathrm{m}$ and smaller micro-pores with the size less than $5 \mu \mathrm{m}$ distributed in larger micro-pores. The CNTs were uniformly distributed in the PU matrix (Fig. S3). These hierarchical micro-pores are conducive to the diffusion of Ag-ion precursor in the next step to form the electrically conductive dual-pathways of 0D AgNPs and 1D CNTs.

To facilitate the electrothermal performance, the AgNPs were introduced to improve the conductivity of the composite fibers further. As shown in Fig. 3a and
Fig. S4, through in-situ chemical reduction of the Ag-ion precursor, the AgNPs were uniformly coated on the surface of micro-pores in the CNT/PU composite fibers. The AgNPs showed a size distribution ranging from 50 to $300 \mathrm{~nm}$ and an average size of ca. $160 \mathrm{~nm}$ (Fig. S4c). The deposition of AgNPs led to the formation of another conductive pathway consisting of $0 \mathrm{D}$ AgNPs. To further discuss the influence of AgNPs and CNTs on the conductivity of fibers, we compared the conductivities of the stretchable conductive fibers with different contents of AgNPs and CNT types. As shown in Fig. S4d, the conductivities of fibers first increased with the increase of AgNP loadings and gradually reached a plateau. Therefore, we selected the AgNP loading of $33.4 \mathrm{wt} \%$ to prepare the stretchable conductive fibers. In addition, the singlewalled CNTs (SWCNTs, diameter: <2 nm; length: 5-10 $\mu \mathrm{m}$ ), another type of CNTs, were used to prepare the porous CNT/PU fibers. Compared with our original CNT/PU fibers using MWCNTs (diameter: $<8 \mathrm{~nm}$; length: $10-30 \mu \mathrm{m})$, SWCNT/PU fibers showed higher conductivity of $1.84 \mathrm{~S} \mathrm{~cm}^{-1}$. However, given that the AgNPs mainly contribute to the conductivity of the conductive fibers, and SWCNTs are relatively expensive, we finally selected the MWCNTs to connect AgNPs to form the electrically conductive dual-pathways. With the conductive dual-pathways of $0 \mathrm{D}$ AgNPs and 1D CNTs, the electrons can quickly transport on the surface and in the polymer matrix of composite fibers, which results in highly improved electrical conductivity of $2100 \mathrm{~S} \mathrm{~cm}^{-1}$. In contrast, the conductivities of CNT/PU and AgNP/PU fibers were lower and calculated to be 0.175 and $1900 \mathrm{~S} \mathrm{~cm}^{-1}$, respectively, further proving the synergistic coupling of interconnected CNTs and AgNPs.

In addition, the formation of electrically conductive dual-pathways can still ensure the electron transport through connective AgNPs and CNTs under the stretching state, thus preventing the sharp decay of con-
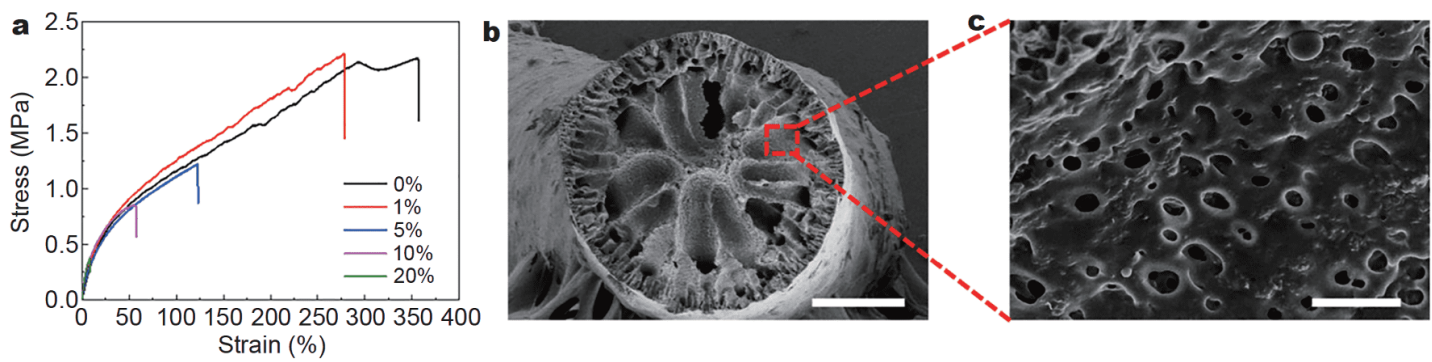

Figure 2 Mechanical performance and morphology of porous CNT/PU composite fibers. (a) Stress-strain curves of the porous CNT/PU composite fibers with different loadings of CNTs. (b) Cross-sectional SEM image of the porous CNT/PU composite fiber (scale bar: $200 \mu \mathrm{m}$ ). (c) Enlarged SEM image of the porous $\mathrm{CNT} / \mathrm{PU}$ composite fiber (scale bar: $5 \mu \mathrm{m}$ ). 

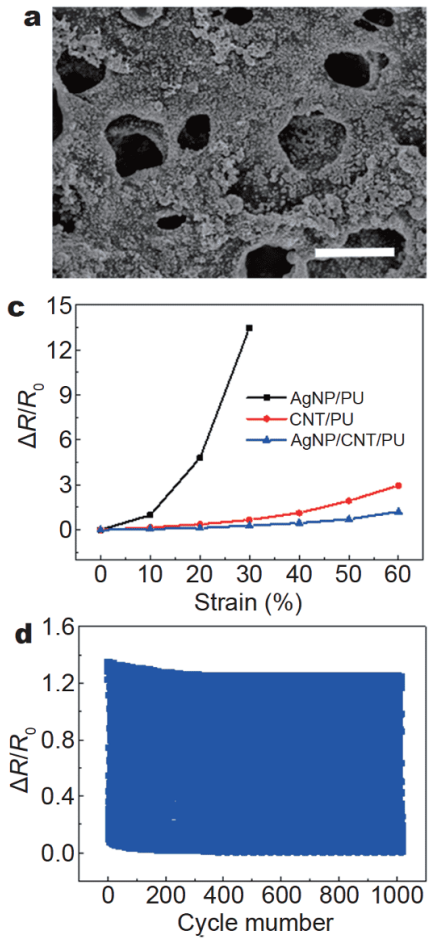

b

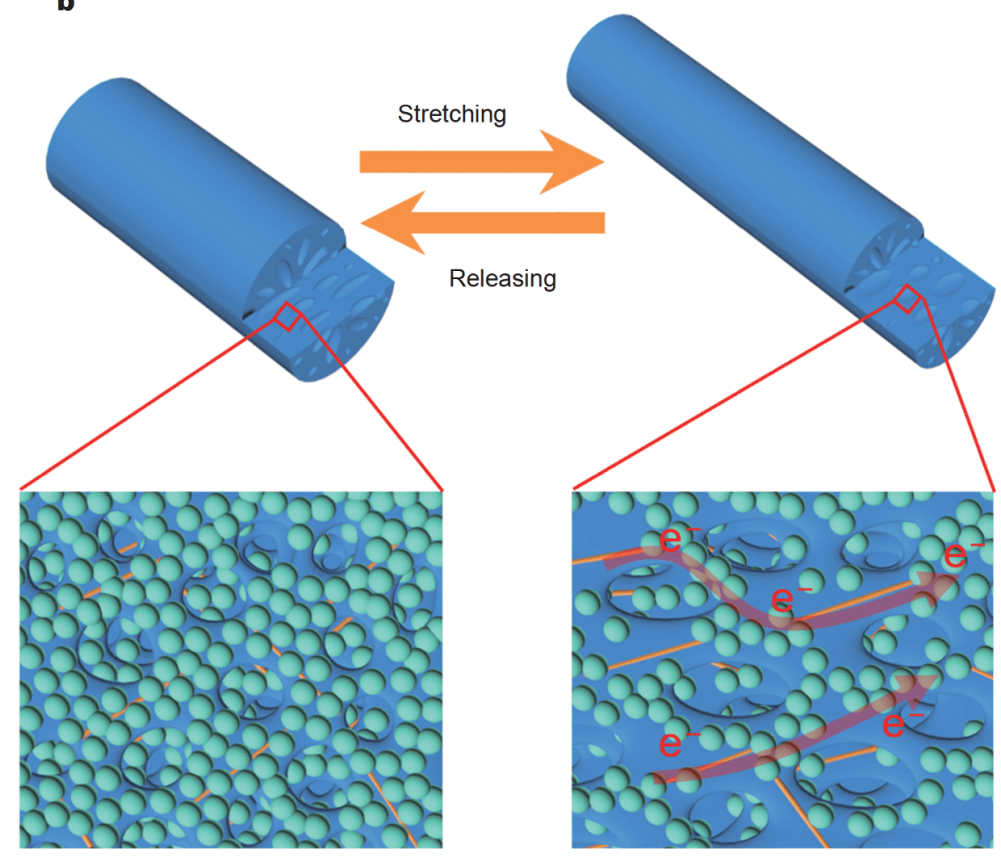

Figure 3 Tensile stability and conductive mechanism of the stretchable conductive fibers. (a) SEM image of the stretchable conductive fiber (scale bar: $3 \mu \mathrm{m}$ ). (b) Schematic diagram of conductive dual-pathways of the stretchable conductive fibers under stretching/releasing states. (c) Resistive ratio $\left(\Delta R / R_{0}\right)$ variations as a function of tensile strains of the stretchable conductive fibers fabricated with different preparation conditions. (d) Cycling stability of the stretchable conductive fiber during repeated stretching/releasing process with the maximum strain of $60 \%$.

ductivity of conductive fibers (as illustrated in Fig. 3b). As shown in Fig. $3 c$ and $d$, the resistive ratio of the stretchable conductive fibers only increased by $\sim 1.5$ folds at $60 \%$ strain and showed great tensile stability after 1000 stretching/releasing cycles (Fig. 3d). In comparison, the resistance of AgNP/PU fiber rapidly increased by $\sim 15$ folds under 30\% strain due to the separated AgNPs under stretching state. The resistive ratios of CNT/PU fibers also increased by 3 folds at $60 \%$ strain due to the disconnection of CNT networks.

Using the conductive fibers with high conductivity and tensile stability as core layers, the stretchable electrothermochromic fibers were realized through coating with various thermochromic inks. As shown in Fig. 4a, under the constant current of ca. $2 \mathrm{~A}$, the stretchable electrothermochromic fibers showed the temperature increase from $19.8^{\circ} \mathrm{C}$ (room temperature) to $27.0^{\circ} \mathrm{C}$. When a $30 \%$ strain was applied, the equilibrium temperature of stretchable electrothermochromic fibers increased from 27.0 to $38.3^{\circ} \mathrm{C}$. The equilibrium temperature further increased to $48.1^{\circ} \mathrm{C}$ as the strain further increased to $60 \%$, due to improved heat generation caused by the increase of resistance. Because of the increased temperature during the stretching process, multiple color changes were exhibited from red to blue and finally to white, and the reflective peak shifted evidently from $\sim 700$ to $\sim 480 \mathrm{~nm}$ at $30 \%$ strain and finally disappeared at $60 \%$ strain (Fig. $4 \mathrm{~b}-\mathrm{d}$ ). Additionally, as shown in Fig. 4e, the $\Delta \mathrm{RT}$ of the stretchable electrothermochromic fibers at the wavelengths of 615 and $524 \mathrm{~nm}$ were tested during 1000 stretching/releasing cycles under maximum stains of $30 \%$ and $60 \%$, respectively, and both almost maintained constant, which proved that the excellent tensile stability of stretchable electrothermochromic fibers. These strain-dependent color changes promise the application of our stretchable electrothermochromic fibers in visual mechanical sensing for smart clothing.

In addition to the strain-induced color changes, the stretchable electrothermochromic fibers also showed controllable color changes by applying different voltages. As shown in Fig. 5a, the equilibrium temperature of the stretchable electrothermochromic fiber increased consistently as the applied voltage increased. Under the voltage of $5.2 \mathrm{~V}$, the equilibrium temperature of fibers reached $38.9^{\circ} \mathrm{C}$ (from room temperature of $20.0^{\circ} \mathrm{C}$ ), leading to the color change of fibers from red to blue. As 

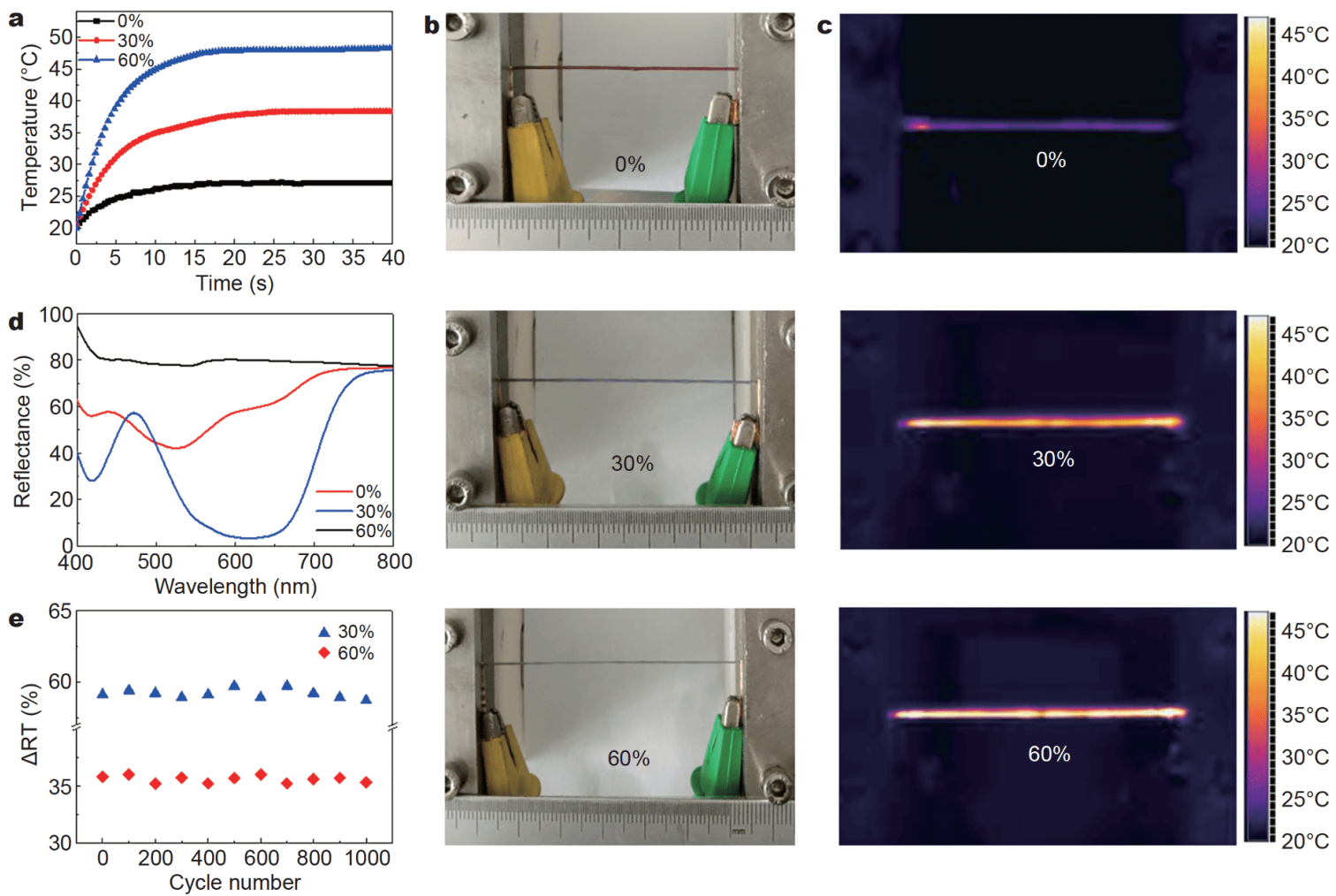

Figure 4 Color-changing performances of the stretchable electrothermochromic fibers under different strains. (a) Temperature-time curves, (b) digital photographs, (c) corresponding thermal images and (d) reflectance spectra of the stretchable electrothermochromic fibers at different stains. (e) Cyclic stability of the stretchable electrothermochromic fibers during repeated stretching/releasing processes under maximum stains of $30 \%$ and $60 \%$, respectively.

the voltage further increased to $7 \mathrm{~V}$, the color of fibers changed to white due to the temperature increase to $49.1^{\circ} \mathrm{C}$. Here, we also measured the switching speeds and cyclic stability of the fibers between red and white. As shown in Fig. 5b, switching times were $9 \mathrm{~s}$ (change to white color) and $10 \mathrm{~s}$ (recover to red color), respectively. The $\Delta \mathrm{RT}$ of the stretchable electrothermochromic fiber was almost unchanged during the 1000 cycles, indicating the great cyclic stability of color changes (Fig. 5c). Contributed by the great flexibility and structural stability, the stretchable electrothermochromic fibers showed multiple color changes under different voltages, even at the knotted state (Fig. S5). The durability of the stretchable electrothermochromic fibers was also measured through a fabric abrasion tester. As shown in Fig. S6, after 500 frictional cycles, the weight of the stretchable electrothermochromic fibers was almost the same, indicating excellent adhesive stability of the thermochromic coatings. As shown in Fig. S7, the reversible color changes of a 2-m-long electrothermochromic fiber were realized under the safety voltage of continuous contact for the human body $(24 \mathrm{~V})$. The stretchable electrothermochromic fiber was also implanted into the textiles and woven into a pattern of "clover". The "clover" pattern showed color changes from red to blue to white under different voltages, promising the potential applications of our stretchable electrothermochromic fibers in the wearable display (Fig. 5d). It should be noted that patterns and color changes can be custom-designed using various thermochromic inks with different color changes or switching temperatures.

\section{CONCLUSIONS}

In summary, the stretchable conductive fibers were first prepared with hierarchical porous structures through the phase inversion of the CNT/PU solution, followed by the deposition of AgNPs. With the formation of electrically conductive dual-pathways consisting of $0 \mathrm{D}$ AgNPs and 1D CNTs in the PU matrix, the stretchable conductive fibers exhibited highly improved conductivity and stable electron transport under the stretching state. In addition, the stretchable electrothermochromic fibers were fabricated with the stretchable conductive fibers as core layers and thermochromic coatings as shell layers. The 

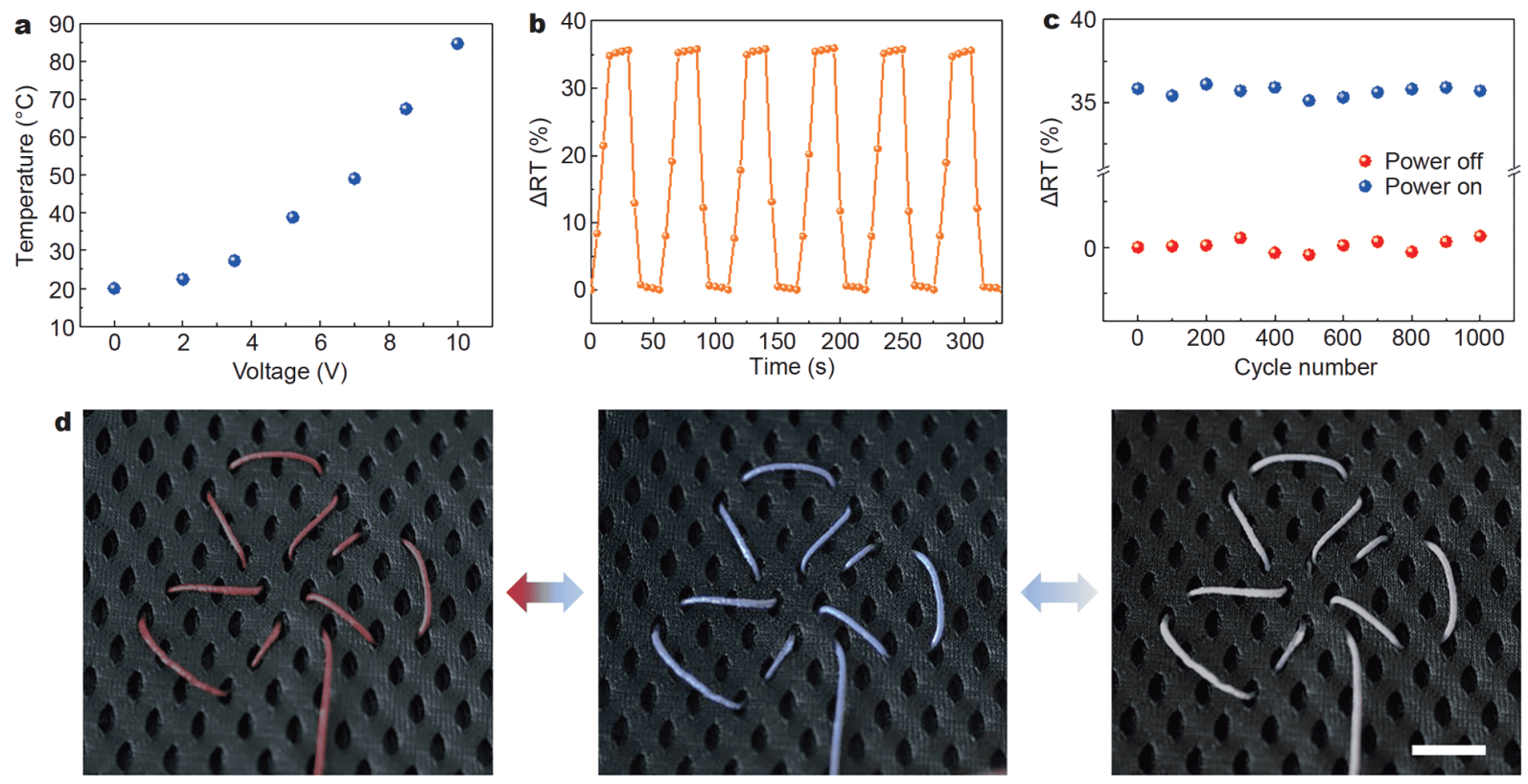

Figure 5 Color-changing performances of the stretchable electrothermochromic fibers under different applied voltages. (a) Temperature changes of the stretchable electrothermochromic fibers under different voltages. (b) In-situ reflectance response of the stretchable electrothermochromic fiber under switched voltages between 0 and $7 \mathrm{~V}$ at wavelength of $524 \mathrm{~nm}$. (c) $\Delta \mathrm{RT}$ of stretchable electrothermochromic fiber at wavelength of $524 \mathrm{~nm}$ during 1000 color-changing cycles. (d) Digital photographs of the stretchable electrothermochromic fiber with pattern of "clover" implanted into textile (scale bar: $3 \mathrm{~mm}$ ).

stretchable electrothermochromic fibers with multiple color changes under different strains or different voltages were able to be implanted into textiles perfectly, advancing their applications in smart clothing.

\section{Received 2 April 2020; accepted 20 May 2020;} published online 29 July 2020

1 House DH, Breen DE. Cloth Modeling and Animation. Boca Raton: AK Peters Ltd./CRC Press, 2000

2 Chae S, Park H, Yoon J, et al. Polydiacetylene supramolecules in electrospun microfibers: Fabrication, micropatterning, and sensor applications. Adv Mater, 2007, 19: 521-524

3 Cai L, Peng Y, Xu J, et al. Temperature regulation in colored infrared-transparent polyethylene textiles. Joule, 2019, 3: 14781486

4 Gao HL, Zhao R, Cui C, et al. Bioinspired hierarchical helical nanocomposite macrofibers based on bacterial cellulose nanofibers. Natl Sci Rev, 2020, 7: 73-83

5 Ma T, Gao HL, Cong HP, et al. A bioinspired interface design for improving the strength and electrical conductivity of graphenebased fibers. Adv Mater, 2018, 30: 1706435

6 Ge J, Sun L, Zhang FR, et al. A stretchable electronic fabric artificial skin with pressure-, lateral strain-, and flexion-sensitive properties. Adv Mater, 2016, 28: 722-728

7 Woo J, Lee H, Yi C, et al. Ultrastretchable helical conductive fibers using percolated Ag nanoparticle networks encapsulated by elastic polymers with high durability in omnidirectional deformations for wearable electronics. Adv Funct Mater, 2020, 1910026
8 Lee S, Shin S, Lee S, et al. Ag nanowire reinforced highly stretchable conductive fibers for wearable electronics. Adv Funct Mater, 2015, 25: 3114-3121

9 Fu X, Li Z, Xu L, et al. Amphiphilic core-sheath structured composite fiber for comprehensively performed supercapacitor. Sci China Mater, 2019, 62: 955-964

$10 \mathrm{Wu} \mathrm{X}$, Peng H. Polymer-based flexible bioelectronics. Sci Bull, 2019, 64: 634-640

11 Hong Y, Cheng XL, Liu GJ, et al. One-step production of continuous supercapacitor fibers for a flexible power textile. Chin J Polym Sci, 2019, 37: 737-743

12 Lu X, Zhang Z, Sun X, et al. Flexible and stretchable chromatic fibers with high sensing reversibility. Chem Sci, 2016, 7: 5113-5117

13 Khudiyev T, Hou C, Stolyarov AM, et al. Sub-micrometer surfacepatterned ribbon fibers and textiles. Adv Mater, 2017, 29: 1605868

14 Jin Y, Lin Y, Kiani A, et al. Materials tactile logic via innervated soft thermochromic elastomers. Nat Commun, 2019, 10: 4187

15 Huang G, Liu L, Wang R, et al. Smart color-changing textile with high contrast based on a single-sided conductive fabric. J Mater Chem C, 2016, 4: 7589-7594

16 Gauvreau B, Guo N, Schicker K, et al. Color-changing and colortunable photonic bandgap fiber textiles. Opt Express, 2008, 16: $15677-15693$

17 Günay M. Eco-Friendly Textile Dyeing and Finishing. Rijeka: InTech, 2013

18 Hunger K. Industrial Dyes: Chemistry, Properties, Applications. Weinheim: Wiley-VCH, 2002

19 Yoon B, Ham DY, Yarimaga O, et al. Inkjet printing of conjugated polymer precursors on paper substrates for colorimetric sensing and flexible electrothermochromic display. Adv Mater, 2011, 23: 
5492-5497

20 Shi R, Lou Z, Chen S, et al. Flexible and transparent capacitive pressure sensor with patterned microstructured composite rubber dielectric for wearable touch keyboard application. Sci China Mater, 2018, 61: 1587-1595

21 Sun X, Zhang J, Lu X, et al. Mechanochromic photonic-crystal fibers based on continuous sheets of aligned carbon nanotubes. Angew Chem Int Ed, 2015, 54: 3630-3634

$22 \mathrm{Pu}$ J, Wang X, Xu R, et al. Highly stretchable microsupercapacitor arrays with honeycomb structures for integrated wearable electronic systems. ACS Nano, 2016, 10: 9306-9315

23 Jia R, Li L, Ai Y, et al. Self-healable wire-shaped supercapacitors with two twisted $\mathrm{NiCo}_{2} \mathrm{O}_{4}$ coated polyvinyl alcohol hydrogel fibers. Sci China Mater, 2018, 61: 254-262

24 Zhou Y, Fang J, Wang H, et al. Multicolor electrochromic fibers with helix-patterned electrodes. Adv Electron Mater, 2018, 4: 1800104

25 Kinashi K, Suzuki T, Yasunaga H, et al. Carrier-assisted dyeing of poly(L-lactic acid) fibers with dispersed photochromic spiropyran dyes. Dyes Pigments, 2017, 145: 444-450

26 Yuan W, Li Q, Zhou N, et al. Structural color fibers directly drawn from colloidal suspensions with controllable optical properties. ACS Appl Mater Interfaces, 2019, 11: 19388-19396

27 Shang S, Liu Z, Zhang Q, et al. Facile fabrication of a magnetically induced structurally colored fiber and its strain-responsive properties. J Mater Chem A, 2015, 3: 11093-11097

28 Pinto TV, Cardoso N, Costa P, et al. Light driven PVDF fibers based on photochromic nanosilica@naphthopyran fabricated by wet spinning. Appl Surf Sci, 2019, 470: 951-958

29 Shang S, Zhang Q, Wang H, et al. Facile fabrication of magnetically responsive PDMS fiber for camouflage. J Colloid Interface Sci, 2016, 483: 11-16

30 Pinto TV, Fernandes DM, Guedes A, et al. Photochromic polypropylene fibers based on UV-responsive silica@phosphomolybdate nanoparticles through melt spinning technology. Chem Eng J, 2018, 350: 856-866

31 Li K, Shao Y, Yan H, et al. Lattice-contraction triggered synchronous electrochromic actuator. Nat Commun, 2018, 9: 4798

32 Li K, Zhang Q, Wang H, et al. Red, green, blue (RGB) electrochromic fibers for the new smart color change fabrics. ACS Appl Mater Interfaces, 2014, 6: 13043-13050

33 Li Q, Li K, Fan H, et al. Reduced graphene oxide functionalized stretchable and multicolor electrothermal chromatic fibers. J Mater Chem C, 2017, 5: 11448-11453

34 Zhou Y, Zhao Y, Fang J, et al. Electrochromic/supercapacitive dual functional fibres. RSC Adv, 2016, 6: 110164

35 Sukitpaneenit P, Chung TS. Molecular elucidation of morphology and mechanical properties of PVDF hollow fiber membranes from aspects of phase inversion, crystallization and rheology. J Membrane Sci, 2009, 340: 192-205
Acknowledgements This work was supported by the National Natural Science Foundation of China (51672043), Donghua University Distinguished Young Professor Program (LZB2019002), Young Elite Scientists Sponsorship Program by China Association for Science and Technology (2017QNRC001), and the Fundamental Research Funds for the Central Universities (CUSF-DH-D-2018006).

Author contributions Li K, Fan H, and Li Q conceived the project; Fan $\mathrm{H}$ and $\mathrm{Li} \mathrm{Q}$ carried out the preparation and measurement of stretchable electrothermochromic fibers; Wang H, Hou C, Zhang Q and Li Y assisted the result analysis; Fan $\mathrm{H}, \mathrm{Li} \mathrm{K}$ and Wang $\mathrm{H}$ co-wrote the manuscript.

Conflict of interest The authors declare that they have no conflict of interest.

Supplementary information Supporting data are available in the online version of the paper.

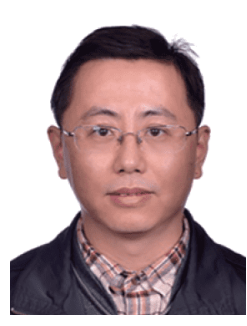

Hongzhi Wang joined the College of Material Science and Engineering in Donghua University as a full professor in 2005. Before that, he completed his postdoc research at the National Institute of Advanced Industrial Science and Technology (AIST), Japan. In recent years, he leads a research group at Donghua University and pursues to construct various flexible multifunctional devices, including flexible optoelectronic devices, artificial muscles and flexible energy sources/systems for smart textiles/clothing.

\section{基于导电双路径分级多孔结构的可拉伸电热致变 色纤维}

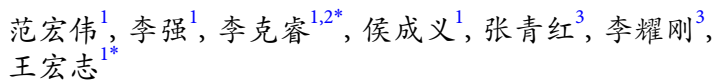

摘要 可拉伸变色纤维具有良好的服装植入性、透气透热性、柔 性和可拉伸性. 因此, 目前对于可拉伸变色纤维的研究受到广泛关 注. 本文以导电纤维为芯层、热致变色涂层为壳层, 制备了可拉伸 电热致变色纤维. 通过相转换法, 在聚氨酯基体中构建了一维导电 碳纳米管(CNTs)网络的分级多孔结构. 通过在微孔表面沉积银纳 米颗粒(AgNPs), 使可拉伸导电纤维内形成AgNPs和CNTs的导电 双路径, 从而有效提高了纤维的电导率和电热性能, 保证了电子的 正常传输, 有效避免了纤维在拉伸状态下电导率的急剧衰减. 此外, 通过连续的湿法纺丝方法可制备长达几米的可拉伸导电纤维. 涂 覆热致变色涂层后, 所制备的电热致变色纤维可完美地植入到织 物中进行多种图案的编织, 并实现多种颜色变化, 在可穿戴显示、 自适应伪装等智能服装领域具有广阔的应用前景. 